\title{
Questes
}

vestes Revue pluridisciplinaire d'études médiévales

$23 \mid 2012$

Le doute

\section{Doute et méthode chez Nicole Oresme}

\section{Sophie Serra}

\section{(2) OpenEdition \\ Journals}

\section{Édition électronique}

URL : http://journals.openedition.org/questes/960

DOI : 10.4000/questes.960

ISSN : 2109-9472

\section{Éditeur}

Les Amis de Questes

\section{Édition imprimée}

Date de publication : 15 mars 2012

Pagination : 48-64

ISSN : 2102-7188

\section{Référence électronique}

Sophie Serra, "Doute et méthode chez Nicole Oresme », Questes [En ligne], 23 | 2012, mis en ligne le

01 janvier 2014, consulté le 30 avril 2019. URL : http://journals.openedition.org/questes/960 ; DOI :

$10.4000 /$ questes. 960 


\section{Doute et méthode chez Nicole Oresme}

\section{Sophie SERRA}

Nicole Oresme n'est pas un penseur du doute. Il faut entendre par là qu'il n'a consacré à cette notion aucun traité spécifique et que, lorsque l'on aborde son œuvre, celle-ci semble dans un premier temps présenter le front uni du rationalisme scientifique davantage que celui, torturé, du scepticisme. Cependant, le doute traverse toute la pensée de Nicole Oresme sous quatre formes, que je présenterai successivement : la superstition, la méthode, le probabilisme, et la faillibilité humaine.

Mais auparavant, revenons brièvement sur la vie et les travaux d'Oresme, ce qui nous permettra également d'aborder un autre des visages du doute : la crainte. Nicole Oresme (1325-1382) commence par étudier la philosophie naturelle avec Jean Buridan à la Faculté des Arts de l'Université de Paris, avant de devenir docteur en théologie, puis d'assumer diverses charges religieuses en Normandie et à Paris, en parallèle avec ses activités à la cour du roi Charles V. C'est cette présence auprès du roi, dont il fut un proche conseiller, qui va lui donner l'occasion de réaliser ses travaux les plus célèbres : aux traités d'économie, de mathématiques, de théologie, de physique déjà composés, viendront s'ajouter en effet plusieurs traités anti-astrologiques, ainsi que les traductions en français de nombreux ouvrages d'Aristote.

C'est à la lecture de ces ouvrages que l'on est amené à mesurer la crainte de Nicole Oresme à l'idée que ses traductions ne soient pas à la fois exactes, éloquentes et techniques. Ainsi, dans le proème de sa traduction des Éthiques d'Aristote, il annonce que, s'il a tâché de traduire avec exactitude le texte d'Aristote, il doit «estre excusé en partie se [il] ne parle en ceste matiere si proprement, si clerement et si ordenement comme il fust 
metier» ${ }^{1}$. À de nombreuses reprises, le philosophe normand souligne le caractère novateur de cette entreprise de traduction, et les approximations qui y sont nécessairement attachées, puisque le français ne possède alors pas encore de vocabulaire technique. Comme nous le verrons par la suite, ce n'est pas seulement le manque d'assurance de Nicole Oresme qu'il faut voir ici, mais également sa conviction que toute activité de recherche est perfectible, et qu'un traité ne doit pas être considéré comme une œuvre finie, mais comme un ouvrage qui demandera dans le futur à être complété et corrigé. C'est en ce sens qu'il n'hésitera pas à ajouter aux exemples de gouvernement qui ouvrent la Politique d'Aristote des exemples tirés de la guerre des Gaules ou même de la guerre de Cent Ans.

Mais la crainte que le langage employé ne soit pas adapté aux thèses examinées ne se limite pas chez Oresme aux langues vernaculaires. Ainsi, dans le proème du De configurationibus, il reconnaît également qu'il n'a peut-être saisi que confusément («confuse sentire») ce qu'il souhaitait exprimer par les mathématiques, qu'il a peut-être parlé de façon obscure («obscure eloqui ») et qu'il n'a pas choisi la meilleure façon de traiter les phénomènes décrits («inconvenienter aptare $»)^{2}$. À travers cette question de l'inadéquation de la forme au fond, on peut pourtant percevoir que c'est moins la crainte de l'incommunicabilité qui hante Oresme que celle de produire des œuvres dépourvues de vie, qui n'inciteront pas les hommes à rechercher à leur tour la vérité. Car s'il a pris le risque d'écrire en français en dépit de l'insuffisance de cette langue, c'est en partant du constat que les hommes de pouvoir auxquels il voulait adresser ses conseils maitrisaient

\footnotetext{
${ }^{1}$ NiCOLE Oresme, Livre de éthiques d'Aristote, Albert D. MenUt (éd.), New York, G.E. Stechert and Co, 1940, 2a, p. 100.

${ }^{2}$ Nicole Oresme and the Medieval Geometry of Qualities and Motions. A Treatise on the Uniformity and Difformity of Intensities known as Tractatus de configurationibus qualitatum et motuum, Marshall ClageTT (éd.), Madison, Londres, The University of Wisconsin Press, «The University of Wisconsin Publications in Medieval Science », 12, 1968, p. 158.
} 
mieux la langue vernaculaire que le latin. Ses textes en français possèdent donc une double fin : parler aux hommes auxquels ils s'adressent, mais également contribuer à amenuiser le risque d'imprécision de la langue. Et afin de parvenir à un équilibre entre ces deux entreprises, il est nécessaire que ces textes soient relus et repris par d'autres. Car il semble bien que ce soit là la seule manière pour la connaissance humaine de perdurer et d'aspirer à l'universalité, les individus s'inscrivant toujours dans une époque, des coutumes et des préjugés contingents. Cette question de l'herméneutique et de la fidélité à l'intention des ouvrages écrits chez Oresme mériterait qu'on lui consacre un examen approfondi. Mais revenons au sujet qui nous occupe pour l'heure.

\section{La méthode}

Face à la question du doute, le Moyen Âge a souvent été qualifié d'«âge de la foi », notamment par Sabina Flanagan dans son ouvrage Doubt in an Age of Faith ${ }^{3}$. Cependant, cette assertion mérite d'être nuancée. Si on l'accepte telle quelle, le doute prendrait sa source la plus éminente dans notre rapport à Dieu : la toute-puissance divine permettrait à celui-ci de nous tromper, ponctuellement (manipulation des causes secondes, c'està-dire interventions miraculeuses), ou bien essentiellement (en nous ayant créés déficients par nature). Le Moyen Âge, s'il a fait grand cas de ces questions, n'a pas vu que des philosophes d'inspiration uniforme y répondre et certains penseurs ont même radicalement reformulé ces problématiques.

En opposition avec le scepticisme absolu, on peut considérer qu'il existe deux attitudes opposées, l'empirisme rationaliste et le fidéisme. Cette typologie représentait déjà, au Moyen Âge, une alternative commune, bien qu'exprimée en d'autres termes. Oresme parvient, lui, de façon très

\footnotetext{
${ }^{3}$ Sabina Flanagan, Doubt in an Age of Faith: Uncertainty in the Long Twelfth Century, Turnhout, Brepols, « Disputatio (Turnhout)», 17, 2008.
} 
cohérente, à briser cette dichotomie.

Son empirisme s'exprime dans l'étude des conditions de formation des gouvernements, dans ses gloses sur la Politique d'Aristote ${ }^{4}$. Oresme énumère en effet différents types de gouvernements à travers de nombreux exemples historiques, comme le faisait Aristote. Et il n'hésite pas à ajouter de nouvelles références, comme s'il s'agissait de passer en revue le plus grand nombre de situations connues, plutôt que de déterminer les causes des configurations politiques particulières. Car le but d'Oresme est de se préparer à faire face à toutes les situations possibles, afin de rendre le philosophe apte à les décrire, et le politique apte à les appréhender s'il les rencontre. Sa méthode, par ailleurs, n'est pas sans évoquer la notion humienne de concomitance (c'est-à-dire l'analyse de plusieurs phénomènes sans faire intervenir de relation de causalité stricte), du moins dans un premier temps de son exposé. Car Oresme décrit différentes configurations qui ont vu naître des cités qui, du fait même de leur existence, représentaient en un lieu et un temps déterminés ce qui pouvait être, et donc ce qui était pour le mieux. Il est peu probable que ces situations singulières se reproduisent à l'identique, étant donné la complexité des phénomènes, et de toute façon, aux yeux d'Oresme, nous ne serions pas capables d'en extraire les principes universels. Ce dernier suppose simplement que les lois positives qui régissent les communautés politiques sont toutes conformes à la nature, puisqu'elles existent, même si elles peuvent être en contradiction les unes avec les autres. Ce n'est plus le moment du doute, car l'on se situe dans la perspective d'une philosophie pratique ; néanmoins, on retrouve là encore le même type de flottement quant à la possibilité d'accéder linéairement à l'universel.

\footnotetext{
${ }^{4}$ Nicole Oresme, Livre de Politiques d'Aristote, Albert D. Menut (éd.), Philadelphia, Transactions of the American Philosophical Society, 60, 1970.
} 
Oresme applique la même méthode dans le Livre de divinacions ${ }^{5}$ : le principe même du livre VIII est «vraie probacion du contraire par experience » et «par induction du temps passé » ${ }^{6}$. Au livre XI, il affirme que les interprètes des songes n'établissent pas aussi bien la vérité que les astronomes, car l'astrologie est « trop forte et trop defaillante ${ }^{7}$ c'est-à-dire trop complexe et trop imparfaite. «Trop forte », cela signifie-t-il tout simplement qu'elle est d'une grande complexité car hasardeuse et boursouflée par des thèses ad hoc rajoutées pour pallier son imperfection? Ou bien qu'elle est trop complexe pour l'homme, dans le sens où elle cherche à embrasser la totalité des phénomènes du cosmos, entreprise rationnelle et légitime, mais réservée à Dieu car au-delà des moyens humains ? Oresme laissera le doute planer sur cette question dans tous ses ouvrages abordant la question, mais il n'est peut-être pas innocent que, justement, presque tous ses ouvrages abordent la question. En tous les cas, même les hommes les plus versés en astronomie divergent dans leurs interprétations, remarque Oresme en laissant au lecteur le soin de conclure. C'est là un premier signe de la dialectique, au sens socratique du terme, mise en place par Oresme dans plusieurs de ses traités.

C'est ainsi que, dans le Livre de divinacions, de nombreux arguments peuvent sembler surprenants, car Oresme utilise comme prémisses des présupposés de l'astrologie qu'il retourne ensuite contre elle pour montrer son inconsistance, mais aussi parce que, s'il en condamne l'emploi, le christianisme ne nie pas l'efficacité de certaines pratiques magiques. S'il ne faut pas se laisser abuser par cette méthode, on peut cependant être surpris de constater qu'Oresme, au chapitre XVI, justifie la prospérité des Romains, qui pratiquaient l'astrologie judiciaire, par le fait «que ilz

\footnotetext{
${ }^{5}$ NiCOLE ORESME, Contro la divinazione, Consigli antiastrologici al re di Francia (1356), Stefano RAPISARDA (éd.), Rome, Carocci, «Biblioteca medievale », 122, 2009. ${ }^{6}$ Ibid., p. 108.

${ }^{7}$ Ibid., p. 130.
} 
faisoient selon l'ordenance des cerimonies de leur religion ou secte $»^{8}$ ! Que peut donc bien signifier ce qu'il conviendrait d'appeler un « relativisme de la providence » sous la plume d'un théologien, au sein d'un traité rationaliste ? Je ne me risquerai pas à sur-interpréter cette thèse surprenante à première vue. Cependant, il semble bien qu'elle trahisse en tous les cas une prudence et un empirisme de la méthode oresmienne plus profonds qu'il n'est communément admis.

À ce propos, il peut être intéressant de considérer l'avis de Marshall Clagett sur la méthode employée par Oresme dans le De configurationibus qualitatum et motuum. Dans ce traité en effet, Oresme se propose de faire correspondre une représentation graphique à la présence de qualités intensives au sein d'une substance. Aux yeux de Marshall Clagett, c'est là une marque du caractère essentiellement conjectural de la méthode oresmienne, dont la doctrine de la configuration n'est autre qu'une « grande ymaginatio ou schéma hypothétique ${ }^{9}$, que le philosophe normand n'hésite pas à manipuler, à mettre à l'épreuve de cas impossibles (comme, par exemple, en tentant de mettre à jour le principe d'action des talismans, dont Oresme nie par ailleurs l'efficacité dans ses écrits anti-astrologiques). Il s'agit avant tout de mettre à l'épreuve les outils élaborés, et de s'assurer qu'ils puissent être utilisés dans l'examen du plus grand nombre de phénomènes ${ }^{10}$.

La méthode oresmienne, comme on le voit, n'est donc pas seulement pragmatique et expérimentale au sens aristotélicien du terme, elle revêt également un caractère provisionnel, pourrait-on dire, qui, bien que surprenant, s'accorde tout à fait avec les convictions profondes d'un

\footnotetext{
${ }^{8}$ Ibid., p. 176.

${ }^{9}$ Nicole Oresme and the Medieval Geometry of Qualities and Motions. A Treatise on the Uniformity and Difformity of Intensities known as Tractatus de configurationibus qualitatum et motuum, éd. cit., p. 12-13, n. 26. Nous traduisons.

${ }^{10}$ C'est-à-dire même si Dieu décidait de changer le cours des évènements de la nature, ou lors d'une intervention miraculeuse particulière.
} 
philosophe qui n'est pas seulement naturaliste, mais également théologien, et garde toujours en tête la possibilité que Dieu puisse modifier le cours naturel des choses, comme nous le verrons.

$\mathrm{Au}$ livre III du Tractatus de commensurabilitate vel incommensurabilitate celi ${ }^{11}$, Oresme se met en scène dans un rêve où Géométrie et Arithmétique débattent sous l'autorité du juge Apollon, lorsque, tout à coup, Apollon suspend le débat. Oresme se trouve alors en proie au doute et s'interroge: les démonstrations des deux partis lui semblent aussi justes l'une que l'autre, et il lui semble tout à coup que leurs arguments n'ont été que rhétoriques et imparfaits. Apollon le rassure : le désaccord entre ces deux mères de la vérité n'est qu'apparent, puisqu'il n'y a qu'une seule vérité, et elles ne faisaient que s'amuser à tourner en dérision l'homme et les méthodes des sciences inférieures. Apollon annonce alors qu'il va permettre à Oresme de sortir de l'incertitude où il était plongé en lui révélant la vérité lorsque, tout à coup, le rêve s'évanouit, et le philosophe, selon ses propres mots, reste seul face à ses doutes ${ }^{12}$.

Le doute est ici présent sous diverses formes, et cette réunion est d'ailleurs surprenante. Tout d'abord, nous sommes dans un rêve, c'est-à-dire dans une situation éminemment subjective et néanmoins universelle, puisqu'alors des vérités sont susceptibles d'être atteintes de manière immédiate, sans passer par les multiples étapes de délibération qui augmentent le risque d'erreur dans la pratique habituelle de la compréhension. Ensuite, nous cherchons à obtenir une réponse à une question dont l'enjeu, justement, est de pouvoir définitivement évacuer par la raison le recours à un art jugé douteux dans plusieurs des traités de

\footnotetext{
11 Nicole Oresme and the Kinematics of Circular Motion. Tractatus de commensurabilitate vel incommensurabilitate motuum celi, Edward GRANT (éd.), Madison, The University of Wisconsin Press, «The University of Wisconsin Publications in Medieval Science », 15, 1971, p. 469-474.

${ }^{12}$ Ibid., éd. cit., III, p. 479-481.
} 
Nicole Oresme, l'astrologie judiciaire. L'auteur rappelle alors à son lecteur qu'il a écrit un ouvrage où les arguments en faveur de l'incommensurabilité sont exposés de manière plus rationnelle et irréfutable encore, le De proportionibus $^{13}$ et, de façon surprenante, ce sont des jugements probabilistes que contient ce traité. Enfin, au sein même de son rêve, Oresme est en proie au doute.

Tout d'abord, il doute de la capacité de la raison humaine à démêler le vrai du faux, en réalisant que les arguments d'Arithmétique et de Géométrie s'opposent mais semblent aussi valables les uns que les autres (Apollon acquiescera d'ailleurs, en disant que, si Oresme est confus, c'est parce qu'elles ont adopté la forme du discours propre aux sciences douteuses des hommes). De plus, le fait même qu'Apollon disparaisse en laissant la question ouverte semble devoir être compris comme une méthode d'exposition efficiente sur le lecteur, une invitation à douter et à trouver la vérité, à s'avancer sur le chemin infini de sa recherche. En effet, Oresme déclare que des éléments de réponse aussi irréfutables qu'il est permis à l'homme doivent pouvoir être reconstruits à partir du discours qu'il a entendu.

\section{La superstition}

Fort de cette possibilité, Oresme écrit son traité De causis mirabilium ${ }^{14}$, afin d'examiner, de remettre en cause, bref, de douter et de résoudre rationnellement des phénomènes perceptifs qui semblent douteux à la plupart des hommes. Il procède à leur examen et cherche à déterminer où il pourrait y avoir rupture du cours habituel des choses. Finalement, ce

\footnotetext{
${ }^{13}$ NiCOLE ORESME, « De proportionibus proportionum » and « Ad pauca respicientes », Edward GRANT (éd.), Madison, The University of Wisconsin Press, « The University of Wisconsin Publications in Medieval Science », 1966.

${ }^{14}$ Nicole Oresme and the Marvels of Nature. A Study of his «De causis mirabilium», Bert Hansen (éd. et trad.), Toronto, Pontifical Institute of Medieval Studies, « Studies and Texts », 68, 1985.
} 
qu'Oresme expose n'est la plupart du temps pas une rupture, mais une autre chaîne causale que celle supposée généralement, plus complexe, et qui bien souvent, une fois reconstituée, semble encore plus invraisemblable (nous dit-il) qu'une explication miraculeuse ou démoniaque.

Il créé donc bien toutes les conditions pour introduire l'incertitude, le doute, voire l'effroi (face à la faillibilité de la condition humaine, face à l'imperfection du monde, etc.), mais, pour autant, garde une certaine confiance en les capacités de la raison. Celle-ci, comme chez Descartes, est responsable de la mauvaise interprétation de nos perceptions sensorielles, mais nous offre en même temps la possibilité, lorsqu'elle est bien utilisée, de remettre les choses en ordre, et cela doit rassurer les hommes.

Lorsqu'Oresme s'attache, dans Le Livre du ciel et du monde ${ }^{15}$, à montrer contre Aristote que tout ce qui a un commencement n'a pas nécessairement une fin, il en profite pour affirmer - momentanément qu'une pluralité de monde est possible (ce genre d'hypothèse est dérivé des condamnations de $1277^{16}$, mais a depuis été une source d'inspiration fructueuse pour l'avancement de la science, et le restera jusqu'au XVII ${ }^{\mathrm{e}}$ siècle). Seulement, il conclue que «même si cela n'était pas le cas en réalité, comme cela ne semble pas l'être d'ailleurs, il [lui] semble qu'il ne serait pas possible d'établir le contraire par un argument logique ${ }^{17}$. La logique, qui constitue pourtant la voie d'accès privilégiée à la vérité scientifique, se révèlerait-elle impuissante à établir ce qui est ?

On remarque deux niveaux différents où le doute peut s'installer. Dans le De causis mirabilium, Oresme affirme qu'il faut chercher des

15 Nicole Oresme, Le Livre du ciel et du monde, Albert Menut et Alexander J. DENOMY (éd.), Madison, The University of Wisconsin Press, «The University of Wisconsin Publications in Medieval Science », 1968.

${ }^{16}$ En 1277, l'évêque de Paris, Étienne Tempier, promulgue un acte de censure contre 219 thèses issues des enseignements donnés à la Faculté des Arts de Paris, et jugées contraires à la foi.

${ }^{17}$ NiCOLE ORESME, Le Livre du ciel et du monde, éd. cit., p. 166. Nous traduisons. 
explications naturelles aux phénomènes apparemment merveilleux, mais que, dans la pratique, il est très difficile d'assigner avec certitude une cause particulière à un effet particulier (car le nombre de causes concomitantes est souvent trop grand pour notre intellect). Il est donc difficile, lorsque l'on est trompé par nos sens, d'établir par nous-mêmes l'explication naturelle de ces phénomènes merveilleux ! D'autre part, dans le De commensurabilitate, c'est la rationalité supposée parfaite du monde lui-même qui se dérobe et peut causer le doute quant à la connaissabilité du monde, non seulement par l'homme, mais en elle-même.

À la fin des Quodlibeta, avant de déclarer que bien des choses en philosophie sont moins connues que de nombreux articles de foi, et que la seule chose dont il est sûr, c'est qu'il ne sait rien, Oresme déclare même que des choses tout aussi miraculeuses sont supposées en philosophie, et sont encore moins démontrées, tout simplement parce que nous y sommes habitués. Pour Edward Grant, c'est là moins l'aveu d'ignorance d'un humble chercheur que l'assertion d'un théologien qui cherche à rabaisser et à décrédibiliser le savoir naturel ${ }^{18}$.

Enfin, dans le De proportionibus proportionum, la méthode oresmienne et sa lutte contre la superstition s'articulent de façon magistrale, nous laissant entrevoir jusqu'où Nicole Oresme est prêt à aller dans sa croisade contre l'astrologie et, surtout, contre les conséquences désastreuses de celle-ci pour les trois domaines auxquels il a dédié sa vie : la politique, la science et la théologie. En effet, en ayant établi par un raisonnement probabiliste que les mouvements des corps célestes étaient incommensurables, Nicole Oresme se défait de la distinction aristotélicienne entre les substances supralunaires et sublunaires. La spécificité des corps célestes étant leur nécessité, celle-ci devait, dans le

18 Edward GRANT, «Jean Buridan and Nicole Oresme on Natural Knowledge », Vivarium, 31, 1 (1993), p. 95. 
cosmos aristotélicien, se répercuter sur leurs mouvements, la parfaite régularité de ceux-ci n'étant autre qu'une conséquence de l'identité de leur essence et de leur fin. En abandonnant cette thèse, Oresme annihile certes la possibilité de l'astrologie judiciaire et ses conséquences funestes sur les comportements humains, celle-ci étant fondée sur la régularité et la cyclicité des mouvements célestes. Mais il abandonne en même temps la conception d'un cosmos parfaitement ordonné par essence et de toute éternité ; or, cette conception avait certes été au cœur de la polémique sur la toute-puissance divine, mais elle garantissait la possibilité d'une connaissance scientifique, d'une connaissance des principes immuables. Privé de cette nécessité, Oresme se voit forcé de reconnaître un caractère approximatif aux sciences physiques, et risque le scepticisme. Ne s'offrent plus alors à ses investigations d'homme de science que les mathématiques.

\section{Le probabilisme}

Oresme rejette la fracture aristotélicienne entre les étants supralunaires, éternels et nécessaires, et les étants sublunaires, non éternels mais qui appartiennent au règne de ce qui se produit le plus souvent.

Ainsi, une conjonction de trois planètes pourrait ne se produire qu'une seule fois en un temps infini. Pour Henri Hugonnard-Roche, c'est précisément dans le but d'établir cela que le philosophe normand aurait mis en place la thèse de l'incommensurabilité ${ }^{19}$. Mais afin de bien comprendre la portée d'une telle affirmation, il serait bienvenu de déterminer ce que Nicole Oresme entend par « possible ».

Dans le sens retenu par Nicole Oresme, «possible» signifie « non-contradictoire » d'un point de vue logique seulement. C'est pour cette

\footnotetext{
${ }^{19}$ Henri HugOnNARD-Roche, «Modalité et argumentation chez Nicole Oresme », in Pierre Souffrin et Alain Philippe SEgOndS (dir.), Nicolas Oresme. Tradition et innovation chez un intellectuel $\mathrm{du} \mathrm{XIV}^{\mathrm{e}}$ siècle, Paris/Padoue, Les Belles Lettres/Programma e 1+1 Editori, « Science et humanisme », 1988, p. 145-163.
} 
raison que dans le Ad pauca respicientes, «possible » est employé au sens de «douteux $»^{20}$. Nous constatons alors que le doute infiltre l'œuvre d'Oresme encore bien plus profondément que nous n'aurions pu le deviner en nous restreignant au seul terme de «doute » et à son acception strictement moderne.

Le philosophe normand estime que le doute est insurmontable quand deux propositions contradictoires sont possibles logiquement. Il est en revanche surmontable lorsqu'on fait appel au probable, c'est-à-dire la faculté d'avancer en faveur d'une proposition des raisons tirées de considérations mathématiques. Seulement, Nicole Oresme prend littéralement ce recours aux mathématiques. Il ne s'agit pas simplement de dire qu'un événement, selon l'expérience sensible, s'est produit le plus souvent, comme en contexte aristotélicien (et comme Nicole Oresme l'affirmait d'ailleurs dans sa traduction de la Politique). Ainsi, les exemples qu'il donne sont ${ }^{21}$ :

- «Le nombre des étoiles est cubique » est improbable car il y a beaucoup moins de nombres cubiques que de non-cubiques.

- Les propositions «Le nombre des étoiles est pair» et «Le nombre des étoiles est impair » ne peuvent pas être départagées par le recours à des raisons mathématiques car il y a autant de nombres pairs qu'impairs. Mais dans ce cas, y a-t-il équipossibilité ? Ou bien cette alternative est-elle laissée ouverte et simplement qualifiée de «douteuse »? Oresme ne tranche pas.

D'où la conclusion d'Henri Hugonnard-Roche :

La probabilité d'Oresme ne concerne pas des évènements ou des classes d'évènements du monde physique susceptibles de se reproduire avec une certaine fréquence. Elle est la transposition directe du résultat d'un calcul combinatoire

\footnotetext{
${ }^{20}$ NiCOLE ORESME, Ad pauca respicientes, éd. cit., I, p. 383-403.

${ }^{21}$ Ibid., I, p. 385.
} 
effectué sur certains objets mathématiques à la distribution des prédicats numériques qui correspondent à ces objets et qui peuvent être attribués à des ensembles d'objets du monde physique. [...] En fait, l'hypothèse de probabilité touchant les mouvements célestes a une fonction essentiellement dialectique; elle sert à réfuter Aristote en fournissant des contre-exemples «imaginaires » aux implications aristotéliciennes, tout en étant elle-même inaccessible à la falsification. ${ }^{22}$

Mais si cette hypothèse est inaccessible à la falsification, elle tombe tout autant que l'astrologie sous l'accusation de pseudo-science qu'Oresme établit dans le Livre de divinacions. Il semble donc qu'aussi ingénieuse que soit cette méthodologie, elle ne soit pas destinée à tromper le lecteur et à instaurer un rapport de force penchant nécessairement du côté de la théologie, mais qu'elle constitue une exploration authentique de la gnoséologie.

Dans la recapitulatio du De causis mirabilium, alors qu'Oresme a produit des démonstrations générales et a souvent adopté un style vif et heuristique, il conclue : "Il a été montré que les causes naturelles ici assignées, et les moyens de les identifier sont possibles et vraisemblables » (« possibilies et verisimiles ») ${ }^{23}$. Il convient donc de remarquer que, d'une part, Oresme semble avoir perdu un peu de l'assurance dont il faisait preuve dans le prologue, aussi bien comme philosophe naturaliste que comme défenseur de la vraie foi. D'autre part, cette mention de la probabilité nous permet de réviser la position peut-être trop stricte selon laquelle la notion de probabilité chez Oresme ne serait jamais expérimentale et naturaliste, mais logico-mathématique, puisque dans la détermination des causes des mirabilia Oresme n'utilise pas ce type d'outils, mais reste très général.

${ }^{22}$ Henri HugOnNARD-Roche, «Modalité et argumentation chez Nicole Oresme », art. cit., p. 158.

${ }^{23}$ Nicole Oresme and the Marvels of Nature, éd. cit., recap. 10-11. Nous traduisons. 


\section{La faillibilité de l'homme}

Dans tous ses ouvrages, Oresme différencie clairement l'astrologie, qu'il combat, et l'astronomie, qu'il considère comme une science noble. Pourtant, toutes deux partagent nombre de principes théoriques, et Oresme ne les différencie jamais plus clairement que lorsqu'il insiste sur les différences qui existent entre leurs pratiques respectives. Alors que, pour faire valoir leurs pseudo-connaissances, les astrologues ont besoin d'affirmer la précision absolue et immuable de leurs observations (afin de dresser des horoscopes, de faire des prévisions portant sur des évènements particuliers), les astronomes véritables, dans leur pratique, doivent sans relâche faire de nouvelles observations pour prendre en compte les approximations de leurs descriptions des positions des corps célestes. En effet, aux yeux d'Oresme, l'exactitude en ce domaine est inatteignable, et c'est même la raison pour laquelle l'astronome est un véritable scientifique, toujours à l'affût de nouveaux phénomènes à observer et comprendre. Nous sommes loin d'une confiance absolue en la précision des sciences naturelles, et pourtant, dans le Ad pauca respicientes, Oresme déclare que ce scepticisme ne doit pas avoir pour conséquence le dédain de l'astronomie :

[Il est] suffisant pour un bon astronome de faire de bonnes estimations des mouvements et des aspects du ciel, du moment que cela n'est pas démenti par ses sens. Mais celui qui cherche davantage, ou pense connaître, travaille en vain. $^{24}$

Il affirme encore, dans le Tractatus de commensurabilitate :

La raison et le rapport de ces choses demeurent occultés de tout temps et ne peuvent être découverts par l'acharnement ou la subtilité d'un homme. Toutefois, cette impossibilité ne produit ni dégoût ni doute, car nous réalisons alors que cela attire et rafraîchit l'esprit, et le pousse à mener plus loin son enquête ; et, avec une suave violence, cela élève les pensées

\footnotetext{
${ }^{24}$ NiCOLE ORESME, Ad pauca respicientes, éd. cit., II, p.428. Nous traduisons.
} 
des mortels. ${ }^{25}$

En effet, selon la devise socratique, citée à plusieurs reprises dans l'œuvre d'Oresme $^{26}$, la connaissance de sa propre ignorance est déjà le témoignage d'une grande sagesse, et le point de départ nécessaire d'une science mesurée et en perpétuelle construction. Oresme va même plus loin puisqu'il poursuit : «De ces choses si excellentes, il serait mieux que quelque chose demeure toujours caché, afin qu'elle puisse être l'objet d'investigations futures $»^{27}$. Car l'état de doute, d'interrogation, est la caractéristique spécifique de l'homme, puisqu'il est à la fois avide de savoir et condamné à toujours ignorer une partie de la vérité. Mais à en croire le vocabulaire utilisé par Oresme (pulcher, excellentia, suavi), cela est beau et réjouissant.

Dans le De causis mirabilium, Oresme indique plusieurs raisons pour lesquelles nous pouvons être victimes d'illusions perceptives : la distance, le milieu, l'imperfection des organes, la faiblesse de notre jugement, mais également la puissance de l'imagination. Celle-ci est bien souvent à mettre en cause, en raison de la crédulité des hommes à l'égard des prédictions des devins. En effet, comme se plaît à le rappeler Oresme à de nombreuses reprises dans ses écrits anti-astrologiques, les prévisions sont souvent vagues et équivoques, et celui qui inclinait déjà à croire en la validité de la divination n'aura aucun mal à interpréter la prédiction reçue comme étant en rapport avec ce qu'il a à l'esprit. Ce ne sont alors pas les faits qui valident la prédiction, mais celui qui désirait croire à sa validité en premier lieu. Aux yeux d'Oresme, le problème ne se situe pas uniquement dans la duplicité des arts occultes, mais dans les conséquences réelles de ces

25 Nicole Oresme and the Kinematics of Circular Motion. Tractatus de commensurabilitate..., éd. cit., prol. 28-36. Nous traduisons.

${ }^{26}$ Par exemple dans les Quodlibeta, IV, Paris, BnF, lat. 15126, fo $98 \mathrm{v}^{\circ}$ : « Ideo quidem nichil scio nisi quia scio me nichil scire. » («C'est pourquoi je ne sais rien, sinon que je sais ne rien savoir $\gg$. Nous traduisons).

27 Nicole Oresme and the Kinematics of Circular Motion. Tractatus de commensurabilitate..., éd. cit., III, 435-446. 
prédictions, distordues par celui qui les reçoit. Car un homme qui penserait que la prédiction lui annonce sa victoire risque d'aller trop sûr de lui à la bataille, de se montrer négligent, et d'être blessé, et un homme qui irait à la bataille en pensant qu'il va être blessé, apeuré et perdant ses moyens, risque tout autant que le premier d'être blessée ${ }^{28}$.

Comme on le voit, ce qui se joue en arrière-plan de la critique de l'astrologie judiciaire ne peut se résumer à une défense de la toute-puissance divine. Il s'agit davantage pour Oresme d'inviter les hommes à cultiver leurs facultés intellectuelles, déjà rudement mises à l'épreuve lors de la recherche de causes complexes, et à ne pas ajouter à la difficulté de la tâche et à la défaillance des sens le pouvoir incontrôlé mais effectif de l'imagination.

Ainsi, nous pouvons conclure avec Edward Grant :

[...] bien qu'il ait entamé notre confiance en notre capacité à déterminer la vérité des causes naturelles, Oresme n'invoque pas Dieu et la théologie pour discréditer les arguments de la philosophie naturelle. Il utilise plutôt sa profonde connaissance de la science aristotélicienne et des mathématiques pour minimiser la revendication de certitude au sein de la philosophie médiévale. Ce faisant, il utilise la raison pour confondre la raison ${ }^{29}$.

Et n'est-ce pas ce que feront les modernes par leurs exercices de pensée sceptique?

Si le doute se rencontre, sous diverses formes, au détour de tous les aspects de la philosophie de Nicole Oresme, il n'apparaît pas pour autant que l'on puisse qualifier sa pensée de «sceptique ». Le doute est parfois un moyen méthodologique de parvenir à ses fins argumentatives, parfois un

\footnotetext{
${ }^{28}$ NiCOLE ORESME, Contro la divinazione..., éd. cit., p. 126.

${ }^{29}$ Edward GRANT, « Nicole Oresme on Certitude in Science and Pseudo-Science », in Pierre SOUFFrIN et Alain Philippe SEgONDS (dir.), Nicolas Oresme. Tradition et innovation chez un intellectuel du XIV é siècle, op. cit., p. 39. Nous traduisons.
} 
moyen de neutraliser les risques de thèses trop audacieuses ou contraires à la foi, mais il ne semble pas non plus, au terme de cette analyse, que l'on puisse réduire cette notion au rôle d'outil, purement extérieur à la pensée d'Oresme. On ne trouve pas chez lui d'arrogance de la certitude absolue et principielle, mais le réconfort d'un usage droit de la raison. Nous n'avons bien sûr passé en revue les textes que d'un seul philosophe médiéval, mais, après cet examen, il semble possible que la thématique du doute au Moyen Âge ne soit pas seulement un héritage amoindri du scepticisme antique, mais une préfiguration de l'usage propédeutique du doute moderne. 